\title{
Privatización Educativa en Brasil: reinventando las Escuelas de Bajo Costo
}

\author{
Cristina Pulido, Ana Ancheta \& Wislleyn \\ Oliveira-Dri
}

\begin{abstract}
Resumen
La educación como negocio como perspectiva de análisis pone de relieve la vulneración del derecho a la educación. El objetivo del siguiente estudio es el análisis del discurso y estrategias de márquetin seguidas para la reciente instalación de las escuelas de bajo costo Luminova, desde finales de 2018, en el estado de São Paulo (Brasil). En concreto, se ha investigado el Grupo Sistema Educativo Brasileño, una entidad financiera y de inversión con carácter multinacional. La metodología que se ha utilizado es la del análisis de contenido cualitativo aplicado sobre noticias de prensa, páginas web del Grupo Sistema Educativo Brasileño y artículos de opinión sobre la multinacional educativa objeto de estudio. En síntesis, se ha comprobado cómo las estrategias de márquetin para instalar las escuelas de bajo costo Luminova en São Paulo son propias del capitalismo creativo y emplean un discurso neoliberal polarizado para destacar los defectos de la oferta pública frente a las virtudes de la privada (nuevas tecnologías, seguridad, inglés e innovación). Finalmente, se alerta de la presencia de estas escuelas como un elemento de competencia directa de la escuela pública que ya convive en un cuasi-mercado educativo existente de manera precedente en el estado paulista brasileño desde la década de los años noventa del siglo XX.
\end{abstract}

Palabras clave:

privatización; escuelas de bajo costo; derecho a la educación; Brasil 


\title{
Educational Privatization in Brazil: Reinventing Low Cost Schools
}

\begin{abstract}
Education as a business as a perspective of analysis highlights the violation of the right to education. The objective of the following study is the discourse analysis and marketing strategies followed for the recent installation of the low-cost Luminova schools, since the end of 2018, in the state of São Paulo (Brazil). Specifically, the Brazilian Educational System Group, a multinational financial and investment entity, has been investigated. The methodology that has been used is the analysis of qualitative content applied to press news, web pages of the Brazilian Educational System Group and articles of opinion on the educational multinational object of study. In summary, it has been proven how the marketing strategies to install the low-cost Luminova schools in São Paulo are typical of creative capitalism and use a polarized neoliberal discourse to highlight the shortcomings of the public offering against the virtues of the private (new technologies, security, English and innovation). Finally, the presence of these schools is seen as an element of direct competition of the public school that already coexists in an educational quasi-market that existed in a previous way in the Brazilian state of São Paulo since the nineties of the 20th century.
\end{abstract}

Keywords: privatization; low-fee private schools; right to education; brazil

\section{Privatização educacional no Brasil: reinventando escolas de baixo custo}

Resumo: A educação como negócio e como perspectiva de análises, coloca em destaque a violação do direito à educação. O objetivo do presente estudo é a análise do discurso e das estratégias de marketing com relação à recente instalação das escolas de baixo custo Luminova, desde o final de 2018, no estado de São Paulo (Brasil). Especificamente, investigou-se o Grupo Sistema Educativo Brasileiro, uma entidade financeira e com investimentos de carácter multinacional. A metodologia utilizada foi a da análise de conteúdo qualitativo aplicada nas notícias da imprensa, das páginas da internet do Grupo Sistema Educativo Brasileiro e dos artigos de opinião sobre a multinacional educativa estudada neste artigo. Nesse sentido, buscou-se mostrar como as estratégias de marketing utilizadas para a divulgação das escolas de baixo custo Luminova em São Paulo são próprias do capitalismo criativo e empregam um discurso neoliberal polarizado para destacar as deficiências da oferta pública em contrapartida as virtudes da oferta privada (novas tecnologias, segurança, inglês e inovação). Portanto, faz-se um alerta sobre a presença de tais escolas como um elemento de concorrência direta com as escolas públicas, que já convivem em um quase-mercado educativo, pois já havia precedentes no estado de São Paulo desde o início dos anos noventa do século XX.

Palavras-chave: privatização; escolas de baixo custo; direito à educação; brasil

\section{Privatisation de l'éducation au Brésil: réinventer les écoles à faible coût.}

Résumé: L'éducation en tant qu'entreprise comme perspective d'analyse qui met en évidence la violation du droit à l'éducation. L'objectif de cette étude est l'analyse du discours et des des stratégies de marketing suivies pour l'installation récente des écoles à faible coût Luminova, depuis fin 2018, dans l'État de São Paulo (Brésil). Plus précisément, le groupe du système éducatif brésilien, une entité financière et d'investissement multinationale, a fait l'objet d'une enquête. La méthodologie qui a été utilisée est l'analyse du contenu qualitatif appliqué aux communiqués de presse, aux sites Web du groupe brésilien du système éducatif et aux articles d'opinion sur l'éducation multinationale, objet de l'étude. En résumé, il a été prouvé que les stratégies de marketing pour installer des écoles à faible coût Luminova À São Paulo, sont les caractéristiques du capitalisme créatif et utilisent un discours néolibéral polarisé pour mettre en évidence les défauts de l'offre publique face aux vertus de l'offre privée (nouvelles technologies, sécurité, anglais et innovation). Pour terminer, nous alertons la présence de ces écoles comme un élément de concurrence directe à l'école publique qui coexiste déjà dans un quasi-marché éducatif existant dans l'État brésilien de São Paulo depuis les années 90 du XXe siècle.

Keywords: privatisation; écoles à faible coût; droit à l'éducation; brésil 


\section{Introducción}

A partir de la década de los 70 del siglo pasado, tras el auge del neoliberalismo, la privatización se establece como un mecanismo, una práctica, una teoría, un discurso y una política aplicada en los diferentes niveles del Estado y que ha transformado el rol que desempeña en la provisión de servicios públicos (Laval y Dardot, 2013).

Moschetti, Fontdevila y Verger (2017) clasifican de manera expositiva la privatización de la educación y sus modalidades de acuerdo con la provisión y financiación de esta de la siguiente manera:

a. Privada-privada: escuelas de titularidad privada, escuelas privadas de bajo costo (low-fee' private schools), clases particulares y escolarización en casa (homeschooling');

b. Privada-pública: cobro de cuotas en escuelas públicas, donaciones filantrópicas a escuelas públicas;

c. Pública-privada: sistemas de voucher, escuelas chárteres y alianzas público-privadas;

d. Pública-pública: escuelas estatales gratuitas.

En ese contexto, se puede exponer que la educación pública en strictu sensu está en peligro y se está convirtiendo en un negocio de mercado en expansión.

Una tendencia de la privatización educativa, señalada en la clasificación anterior, son las escuelas de bajo costo (EBC, en adelante), sobre las que se ha investigado durante la última década (Balarin, 2016; Moschetti, 2015). De hecho, las evidencias demuestran que la proliferación de las EBC se da en los países de bajos y medios ingresos donde se localizan fuertes déficits de oferta educativa estatal. Así, las actividades empresariales han encontrado un fértil campo para cubrir la demanda educativa necesaria. Esta modalidad de negocio se puede mapear en África, Asia y América Latina (Moschetti et al., 2017).

Empero, no se puede hablar de dos realidades iguales ya que las EBC están siendo adaptadas en diferentes territorios. En algunos contextos, están siendo introducidas mediante asociaciones público-privadas, frente a otras formas que parten directamente del establecimiento de una oferta educativa privada que el Estado deja abierta cuando no tiene medios o voluntad para establecer una red de educación pública suficiente (Srivastava, 2015). Otras realidades como las de Reino Unido o Brasil se centran en la provisión de una oferta educativa que compita en los cuasi-mercados educativos de ambos países y aseguren un nuevo mercado que ha demostrado ser beneficioso para las corporaciones que tienen experiencia en otros países (GCE, 2016). 
Este modelo de privatización ha supuesto un giro conceptual si atendemos al hecho de que sus principales clientes son la población de bajos o medios ingresos. Por eso, surgen como una oportunidad para que las empresas educativas puedan establecer nuevos clientes en el negocio de la educación. Hasta comienzos del siglo XXI las familias de bajos y medios ingresos no estaban presentes en el mercado de la escolarización privada (Moschetti, 2015).

Uno de los puntos fuertes de la proliferación de las EBC es que son enunciadas como la única oportunidad para que los pobres tengan acceso a una educación de calidad e innovadora que el Estado no es capaz de ofertar (Moschetti et al., 2017). Según Ball y Olmedo (2011) el discurso que une el nexo entre el ánimo de lucro y "hacer el bien" forma parte de lo que se denomina como "capitalismo creativo".

En un estudio comparado realizado por Tooley y Dixon (2005) entre escuelas públicas y EBC en India, Ghana, Nigeria y Kenya financiado por el Cato Institute - un think tank ${ }^{1}$ neoliberal - afirmaron que: los alumnos obtenían mejores resultados en los exámenes, el profesorado presentaba menores índices de absentismo, había una menor ratio alumno-profesor y contaban con mejores infraestructuras. Si bien, existe una multiplicidad de investigadores que alegan todo lo contrario como, por ejemplo:

- Srivastava (2015) constata que en el caso de las familias de hogares en riesgo no pueden asumir las "bajas" cuotas para acceder a estas escuelas;

- Härma (2011) confirmaba, en la línea anterior, que los dos quintiles de población más pobre no podían acceder a estas escuelas en India;

- Klees (2017), por su parte, analiza el caso de las asociaciones público-privadas con empresas de EBC en Liberia, determinando cómo este programa había supuesto el doble del presupuesto público, en lugar de ser invertido invertirlo en la red de escuela pública y prevenir su abandono;

- Afridi (2017) que estudió las condiciones laborales y de contratación del profesorado en EBC de Punjab (India), destaca que se contrataba mano de obra barata e infraformada priorizando la contratación de mujeres, ya que se encuentran más desprotegidas en este sentido.

Las EBC se están convirtiendo en un producto atractivo para parte de la población allí donde son instaladas, ya que presentan infraestructuras mejoradas, una propuesta curricular que valora los saberes instrumentales demandados por el mercado laboral (matemáticas, lengua y ciencias), apoyan sus actividades en las nuevas tecnologías, presentan modelos pedagógicos basados en la innovación y, en algunos casos, evalúan sus actividades haciéndolas públicas posteriormente para competir (GCE, 2016).

De todo ello, se confirma la importancia y necesidad de estudiar estos modelos de escuelas en sus contextos, para identificar cómo las actividades comerciales y el rol del Estado han permitido hacer de la educación un negocio. En este sentido, la educación como negocio es una perspectiva de análisis interesante que ayuda a denunciar la 
vulneración del derecho a la educación pública. Bajo dicho enfoque, el objetivo principal de este estudio es el análisis de la instalación reciente y novedosa de las EBC como iniciativa de una corporación en el contexto brasileño desde finales de 2018. Para lo que planteamos la siguiente cuestión: ¿puede ser este caso considerado como otro ejemplo de las llamadas EBC conforme a la literatura especializada? En concreto, se investiga la intervención del Grupo Sistema Educativo Brasileño (GSEB, en adelante), una entidad financiera y de inversión que encabeza actividades empresariales en educación en este país latinoamericano. En septiembre de 2018, el GSEB lanzó la publicidad del modelo piloto de EBC denominadas Escolas Luminova que empezarían sus actividades educativas en 2019 con 4 nuevas escuelas en el estado de Sao Paulo (Mello, 2018).

Las EBC ya han sido denunciadas como un modelo de comercialización que juega con las ilusiones de los pobres (Balarin, 2016) y que vulnera el derecho a la educación produciendo beneficios privados y pérdidas públicas; es decir, que producen un alto impacto social polarizando a los ciudadanos cada vez más por su estatus socioeconómico y condición de género (GCE, 2016). Por este hecho, hemos analizado el discurso, las estrategias, la audiencia o a quiénes se dirigen y los empresarios que hay detrás de este modelo de negocio en el caso de Brasil, para denunciar un modelo de actividad económica que exacerba las desigualdades y socava el derecho a la educación de los más pobres.

Para ello, las fuentes primarias y secundarias seleccionadas han sido: la Constitución Federal Brasileña de 1988 (http://www.planalto.gov.br/ccivil_03/Constituicao/ Constituicao.htm) la Ley de Directrices y Bases de la Educación Brasileña de 1996 (http://www. planalto.gov.br/ccivil_03/Leis/L9394.htm) noticias de la prensa brasileña; las plataformas web de la corporación, los materiales de márquetin, así como el material audiovisual del GSEB. Los documentos han sido la base de las argumentaciones y de los análisis realizados en relación con las estrategias, las prácticas y los discursos del GSEB para instalar el modelo de EBC Luminova en Brasil.

La metodología utilizada es la del análisis cualitativo de contenido, es decir, la recogida de información sistemática que nos permite analizarla y comprobar las teorías sociológicas y contextuales a partir de esta para, luego, analizarla y elaborar (o comprobar) alguna teoría o generalización sociológica sobre ella (Minayo y Costa, 2018). A lo largo del artículo, mediante los debates y reflexiones planteados hemos buscado identificar e interpretar lo tácito y lo oculto (Krippendorf, 1997) en el doble discurso de la oferta de un servicio privado como si se tratara de un derecho público que queda supeditado a la capacidad económica de la audiencia a la que se dirige. A su vez, ponemos de relieve la polarización en el discurso del modelo de escuela anunciado, ya que se destacan las virtudes de la escuela privada, frente a una idea de baja calidad de la escuela pública como una estrategia de márquetin empresarial propia de la ideología neoliberal. 


\section{Algunos apuntes sobre la privatización de la educación en Brasil}

La Ley de Directrices y Bases de la Educación Brasileña de 1996 (LDB) y la CF (1988) son las leyes principales que permitieron el crecimiento de la red de educación privada, público-privada y pública. Tal y como se puede observar en la siguiente tabla:

Tabla I. Reglamentaciones que permite la privatización de la educación en Brasil

\begin{tabular}{|c|c|c|}
\hline CF & 1988 & $\begin{array}{l}\text {-Educación como derecho del ciudadano y deber del estado y de la familia } \\
\text { (Art. 205); } \\
\text {-Principio de gratuidad a la enseñanza pública (Art 206); } \\
\text {-La enseñanza es libre a la iniciativa privada (Art. 209); } \\
\text {-La Unión, los estados, el distrito federal y los municipios organizaron en } \\
\text { régimen de colaboración sus sistemas de enseñanza (Art. 211). }\end{array}$ \\
\hline LDB & 1996 & $\begin{array}{l}\text { - Las instituciones de enseñanza de los diferentes niveles se clasifican en las } \\
\text { siguientes categorías administrativas: } \\
\text { I - públicas, así entendidas las creadas o incorporadas, mantenidas y } \\
\text { administradas por el Poder Público; } \\
\text { II - privadas, así entendidas las mantenidas y administradas por personas } \\
\text { físicas o jurídicas de derecho privado (Art. 19). } \\
\text {-Las instituciones privadas de enseñanza se encuadran en las siguientes } \\
\text { categorías: } \\
\text { I - particulares en sentido estricto (...); } \\
\text { II comunitarias, (...) sin ánimo de lucro, que incluyan en su entidad mantenedor } \\
\text { a representantes de la comunidad; } \\
\text { III - confesionales, (...) orientación confesional e ideologías específicas (...); } \\
\text { IV - filantrópicas (...) (Art. 20). }\end{array}$ \\
\hline
\end{tabular}

Fuente: Elaboración propia a partir de la CF (1988) y la LDB (1996).

Las encuestas muestran que la matrícula de educación básica en el sector privado ha aumentado en los últimos años, según Adrião (2018):

(...) entre 2000 y 2015, las matrículas privadas en la educación básica crecieron del 13,97\%, en 2000 al 21,10\%, en 2015. Tal crecimiento viene acompañado de la disminución de la proporción entre el número de establecimientos públicos y privados: en el año 2000 , uno de cada seis establecimientos era privado, en 2015 esa proporción pasó a uno de cada cuatro (p. 15).

Esta realidad viene explicada por la creencia meritocrática instaurada en la sociedad brasileña, que impulsa la idea de que sólo con esfuerzo lograrán un cambio social en contrapartida a una idea de políticas sociales. Esta concepción meritocrática de la educación concomitante a una idea de que la educación pública es de baja calidad y, también, a la mejora de las condiciones económicas de una parcela de las clases 
populares en la última década en el país, ha hecho que muchas familias elijan las escuelas privadas y sacrifiquen gran parte de su renta.

Datos de la misma investigación revelan que: poco más del $50 \%$ de esas escuelas tenían bibliotecas; el 21\% tenía laboratorios de ciencias; más del 55\% no tenían zona deportiva; el 26\% no tenían tratamiento de aguas residuales Adriâo (2018, p.15); y los salarios medios de los profesores de las escuelas privadas eran menores que la media salarial de la red pública. A su vez se destaca que el objetivo de estas escuelas es el lucro que vulnera el derecho a la educación. A su vez, describe dos tipos de escuelas privadas: las EBC de las periferias urbanas y las escuelas de élite para un segmento reducido de la población, produciendo un sistema educativo de dos velocidades.

Todos estos factores, entre otros, permiten o facilitan la entrada del sector privado en la provisión de la educación. Así las EBC en Brasil se muestran como un mercado emergente que es iniciado mediante un estudio previo por parte de las grandes corporaciones, como veremos más adelante.

\section{La emergencia de las Escuelas de Bajo Costo en Brasil: el Grupo Sistema Educativo Brasileño y las Escuelas Luminova en el Estado de São Paulo}

Brasil es un país grande y poblado, estructurado en una República Federal, donde existen grandes diferencias socioeconómicas entre los estados y municipios. No es casual que la corporación estudiada en el presente trabajo haya decidido impulsar el modelo de EBCs en São Paulo, ya que es uno de los estados brasileños más ricos de un total de 27 que conforman el país. Una estrategia que es enunciada así por Tahim Zaher (directora ejecutiva de GSEB) sobre la iniciativa de EBC: "Hemos decidido comenzar por São Paulo porque es donde hay mucha demanda (...)” (DCl, 2018).

El Estado de São Paulo cuenta con más de 45 millones de habitantes que representan el $22 \%$ de la población brasileña (IBGE, 2018). Asimismo, concentra la mayor producción industrial del país y cuenta con importantes centros financieros y corporativos, varias universidades, centros tecnológicos y de investigación, e importantes aeropuertos y carreteras. Fruto de todo ello, ostenta el mayor Producto Interno Bruto (PIB) (https://cidades.ibge.gov.br/brasil/sp/sao-paulo/pesquisa/38/47001?tipo=ranking ) del país. Entre su población se haya una élite dominante, pero también una clase media trabajadora que protagoniza la gran producción de riqueza del Estado. Debido a esto, resulta fácil identificar una clase media que tiene posibilidades financieras de consumir la oferta de las EBC Luminova del GSEB.

En Brasil existen otras empresas o corporaciones que vienen actuando en el campo de la educación. Adrião et al. (2016) realizan una investigación sobre los cinco grupos empresariales en educación más destacados en el estado de São Paulo (Grupo Objetivo, Pearson, Grupo Positivo, Grupo Santillana y Somos Educação), pero desde 
la perspectiva de las actividades educativas en lo público en forma de asociación público-privada, que confirma la presencia de una industria empresarial educativa asentada y prolífica en el Estado paulista.

A la vez, es importante destacar que las EBC existen en el contexto brasileño desde hace ya algunas décadas según Siqueira y Nogueira (2017). Las EBC descritas por estas autoras son propiedad de personas de la región y cuentan con: una estructura física precaria, patrimonio restringido, recursos humanos mínimos, y una mayor interacción de las familias en las escuelas. Ahora bien, lo que las diferencia precisamente de las EBC Luminova, es que éstas últimas forman parte de grandes corporaciones y cuentan con un mayor capital inversor. A la vez, tienen estrategias de márquetin para atraer clientes y cobran cuotas mensuales, es decir, que estarían enmarcadas dentro de iniciativas comerciales, frente a iniciativas filantrópicas como puedan ser las EBC descritas por las autoras.

El estudio de este trabajo se ha centrado en la emergencia del modelo de EBC en el contexto brasileño como iniciativa de una de las mayores cadenas de escuelas privadas en Brasil que cuenta hoy con más de 130.000 estudiantes, es decir, el GSEB (GSEB, 2019). Para comenzar, la propia denominación de la empresa corporativa (GSEB), evoca lo que en Brasil es conocido por el sistema educativo brasileño público, es decir, que usan las mismas siglas para denominar a un grupo empresarial con ánimo de lucro que se presenta como la oportunidad de ofrecer una educación de calidad.

EI GSEB está entre los mayores grupos privados de educación de Brasil y cuenta con una historia de poco más de 50 años que nace en el estado de São Paulo donde, como se ha mencionado de manera previa, están los posibles mayores consumidores de educación privada. En ese sentido, el antiguo Curso Oswaldo Cruz (COC), el antecedente del GSEB, se ha dado a conocer entre los paulistas por su editorial, sus escuelas de educación básica y superior y sus cursos de preparación para los exámenes de ingreso a la Universidad (GSEB, 2019).

EI GSEB ha realizado varias estrategias mercantiles: en 2007 entró en el mercado abierto y en la bolsa de valores; en 2010 vendió parte de su programa tecnológico de enseñanza en inglés a Pearson (previniendo que era el grupo corporativo más fuerte en ese campo y que estaban abocados a desaparecer). A partir de aquí, aumentó su capital y expandieron el negocio por otras zonas de Brasil (más allá de São Paulo). En 2013, realizó un estudio de mercado que les permiten percibir que el crecimiento del negocio estaría en la educación básica y focaliza ahí todas sus actividades, dejando el campo comercial de la educación superior en un segundo plano. Desde esta iniciativa, hicieron "turismo educativo" en busca de sistemas educativos internacionales de éxito para ofertar una educación "basada en evidencias", a lo largo del continente americano y europeo. 
EI GSEB alcanzaría su expansión mundial con la adquisición del 95\% de las operaciones canadienses de Maple Bear en Brasil y una participación del 50\% en la compañía estadounidense Digital Media Academy (BayBrazil, 2017). En 2018 se reinventan $y$, en su lectura del nicho de mercado que ocupan las EBC, ofrecen educación a la clase C (media-baja), tal y como describe Chaim Zaher: "Vamos a atender a la clase C que tiene potencial de consumo y está mal atendida por la esfera pública” (DCl, 2018). En relación con esto, Moschetti et al. (2017, p. 37), describen que el fenómeno de las EBC en Brasil orientado a la población más pobre no ha sido mapeado, ya que según el estudio de Guerra Esteves de Moraes y Belluzzo (2014) referenciado por estos mismos autores, el acceso a la educación privada en un contexto tan desigual como el brasileño, sólo estaría al alcance de la mano de las familias de clase alta y media-alta.

Por ello, nos encontramos ante un nuevo fenómeno en el que el GSEB ha lanzado su propuesta de EBC con una estrategia de márquetin presentándola como un "derecho" de la clase trabajadora. Tal y como se puede observar en su plataforma ${ }^{2}$ y video promocional (GSEB, 2019), se da aquello que Ball y Olmedo (2011) describen como el nexo del "lucro" y "hacer el bien" propios del capitalismo creativo para expandir sus actividades comerciales hacia estratos de la población a los que no habían llegado hasta el momento.

Como se ha descrito de manera previa, la iniciativa del lanzamiento y divulgación de las escuelas Luminova fue en septiembre de 2018 con ubicación en el estado de São Paulo para ofrecer así, como definiría el presidente del grupo empresarial, la oportunidad de una educación de calidad y de bajo coste para los ciudadanos de clase media para competir con las escuelas públicas (DCl, 2018).

Chaim Zaher, describe todas sus iniciativas empresariales como "actividades filantrópicas para acabar con el sistema educativo público K-12 de baja calidad y carente de medios para dotar de una educación de calidad" (BayBrazil, 2017, traducción propia). Así, se trata de una actividad presentada como filantrópica y parte de la lógica neoliberal por la que las escuelas públicas son de baja calidad y es el empresario mediante una iniciativa privada el que puede ofrecer una educación para el siglo XXI (nuevas tecnologías, aprendizaje mediante nuevas metodologías, el profesorado como orientador, etc.), propio del discurso del capitalismo creativo (Ball y Olmedo, 2011).

Se puede observar, igualmente, en la página web de las EBC Luminova, como exponen su propuesta pedagógica concentrada en proporcionar a sus alumnos lo que ellos llaman una "metodología de enseñanza innovadora direccionada por los resultados", destacando el uso de las tecnologías y la enseñanza diaria del inglés. Así, el modelo o producto ofertado en sus páginas web utiliza la herramienta discursiva de la polarización, destacando las virtudes de un sistema educativo moderno, frente al arcaico y saturado modelo educativo público. Las propuestas son lanzadas con aires de innovación, centradas fundamentalmente en el modelo globalizado de educación, 
es decir, la instrumentalización del currículum "matemáticas, ciencias e inglés" y el uso de tecnologías como sinónimo de innovación, así como la rendición de cuentas, evaluación y publicación de sus resultados como índices de calidad educativa (Sahlberg, 2012). El empresario Chaim Zaher, en una entrevista concedida a Bay Brazil (2017, traducción propia) describiría que:

La tecnología es parte de nuestro ADN y, como tenemos el compromiso de brindar la mejor experiencia a nuestros estudiantes y la excelencia académica, la tecnología es una parte esencial de eso. Creemos que la tecnología puede mejorar la enseñanza y el proceso de aprendizaje y proporcionar una experiencia y excelencia optimizadas para los estudiantes (BayBrazil, 2017, párrafo 5)

En su propuesta de EBC, en la página web de Luminova, también destacan otros dos puntos relacionados, el primero con la seguridad del alumnado y el segundo con el profesor influyente. En relación con la seguridad, se propone que todos los espacios de aprendizaje estén vigilados por cámaras de seguridad, siendo una escuela que preserva las relaciones sociales no permitiendo el bullying. Sugieren a las familias una escuela segura en tiempos de violencia, una característica generada por la desigualdad social característica de los países de bajos y medios ingresos. Es decir, establecen su estrategia de márquetin a partir de un problema real y que afecta de forma degradante a las personas en el que el derecho a la seguridad se transforma en un elemento de mercado. La relación entre políticas de mercado y seguridad ante la violencia han sido desarrolladas desde las agendas neoconservadoras, en las que esta unión de principios ha servido de estrategia política para introducir el neoliberalismo, con sus ejemplos más paradigmáticos en Reino Unido y Estados Unidos en la década de los setenta del siglo pasado (Laval et al., 2013).

Otro punto destacado de la estrategia comercial en la página web de Luminova del GSEB es la demarcación del denominado "profesor influyente", donde la empresa educativa justifica que tiene un equipo comprometido con el aprendizaje y la formación integral del alumnado, frente a la figura del profesorado público como una de las figuras más desprestigiadas desde la ideología neoliberal (Luengo y Saura, 2013). Por lo que el GSEB ha construido una imagen de especialistas educativos, mediante el uso de un discurso polarizado en el que destacan sus facultades frente a las críticas a lo público y sus agentes.

Ahora bien, la formación integral de los alumnos es uno de los objetivos de la educación brasileña y está recogida en la CF (1988) y en la LDB (1996). En concreto, no se aporta nada nuevo en su "propuesta pedagógica", lo que se pone de relieve es una oferta más práctica, es decir, utilitarista, poniendo el foco en el mercado de trabajo, promoviendo el ideal de un ciudadano que es pragmático, flexible y que sabe trabajar 
en equipo. Es decir, la figura del profesional en las organizaciones pos-fordistas que, según Hargreaves (1999), termina produciendo profesionales irreflexivos que no invitan a desarrollar en el alumnado un pensamiento crítico al servicio de la construcción de sociedades más justas.

La tecnología, la innovación, la instrumentalización del currículum y la profesionalización de los equipos de trabajo forman parte de la estrategia de márquetin de una cadena o corporación educativa para captar clientes, y, por ende, de lo que se espera de la formación en la era del nuevo capitalismo (GCE, 2016). Las EBC Luminova, presentadas para la clase media y baja, acentúan las posibilidades de elección y de establecer una competencia entre escuelas públicas y privadas, así como una homogeneidad en las distintas redes de escolarización pública, público-privada y privada por niveles socioeconómicos similares.

En el caso de Brasil, estas EBC no surgen de la carencia de oferta educativa por parte del Estado, sino de la apertura del Estado al mercado como proveedor de educación y de la localización de las necesidades de orden instrumental de la clase media para diferenciarse de las personas de su misma clase o clase más baja como una estrategia llevada a cabo por los individuos propia de la cultura del nuevo capitalismo (Olmedo y Santa Cruz, 2008).

\section{Conclusiones}

A grandes rasgos, en el trabajo se ha identificado cómo el GSEB se ha instalado en Brasil, empezando por el discurso, una vez que las siglas del grupo SEB en sí, ya preocupan, pues se definen como el Sistema Educativo Brasileño lo que lleva a pensar ¿Esto es intencionado o es una declaración de futuro? Se puede asegurar que el texto indicó algunas respuestas a esta pregunta y generó, cuanto menos, otras indagaciones sobre el polémico y largo tema de la privatización educativa. Es sabido que la educación se ha convertido en un campo atravesado por especialistas de otros campos (periodistas, médicos, abogados) e incluso de grandes empresarios, ya que la educación es una "preocupación" de todos (aunque con diferentes intereses) y esto la convierte en algo susceptiblemente comerciable.

Por medio de las noticias de la prensa brasileña, las plataformas web de la corporación, los materiales de márquetin y vídeos del GSEB, se pueden percibir relevantes estrategias para captar clientes, que no son los que tradicionalmente consumen la educación privada en Brasil. La promesa de las EBC Luminova es que son "buenas y baratas", y esto ha conducido a plantear que, tras toda la conocida complejidad de la educación y, sobre todo, en contextos tan desiguales como es el caso de Brasil: ¿en detrimento de qué se puede ofertar una educación de buena calidad y de bajo costo? Esta es una pregunta que queda abierta a futuras investigaciones. 
La publicidad del GSEB es muy semejante a las otras cadenas de EBC, pues hay una propuesta pedagógica según lo que demanda el mercado, o sea, el alumnado es "entrenado", las nuevas tecnologías son pieza angular de la propuesta pedagógica, el inglés y las matemáticas están en el centro del proyecto educativo, siendo el modelo que marcan las evaluaciones internacionales de rendimiento y cumpliendo una agenda global (CME, 2019; Sahlberg, 2012). La idea central de estos negocios educativos no es la de educar, sino la de formar para un mercado que promete el ascenso social, pero que en la realidad no necesita un ciudadano crítico y con profundidad en sus reflexiones, sino uno que sepa manejar las nuevas tecnologías a fin de seguir nutriendo una clase trabajadora que genera más ganancias y lucro a los gigantes del mercado (GCE, 2016).

De manera que se ha podido analizar la reinvención de una empresa educativa explotando los distintos nichos del mercado educativo. La propuesta educativa de un modelo privado para las clases medias y las bajas, en la cual se está resignificando el sentido del cliente de élite y permite establecer una red que segrega según las tarifas de pago que establecen, es decir, el trabajador también puede "elegir" una educación privada, aunque esta no sea la misma que la de los hijos del patrón.

En este sentido, la educación pública se ve empujada a "reinventarse o morir" en el mercado educativo en el que las EBC se convierten en una competencia directa, por no mencionar las escuelas público-privadas y otro tipo de escuelas fundacionales que suponen modos de privatización de lo público que han sido estudiadas en Brasil desde otras perspectivas (Adrião, 2018; Adrião et al., 2016; entre otros).

De manera más extrema, y tratando de establecer una generalización sociológica tras el análisis cualitativo del contenido (Krippendorf,1997) pensamos que el futuro de la educación pública puede quedar relegado a la atención de los más pobres que serán aquellos que no podrán pagar por una educación privada, ya que el cuasi-mercado establecido en São Paulo, junto con la aparición de las EBC, puede derivar en lo que ha sido comprobado en otros territorios, es decir, en una mayor segregación e inequidad social (CME, 2019).

Finalmente, explotamos una línea de investigación contemporánea que está ocupando gran parte de los estudios sobre privatización en contextos de países de bajos y medios ingresos. Una vez comprobada la capacidad de las EBC como negocio educativo en los contextos descritos, comienzan a rastrearse en otros países y realidades de altos ingresos, como es en el caso de Inglaterra o Estados Unidos. De este modo, la diferencia principal, entre las EBC que forman parte de iniciativas populares o entidades de beneficencia y empresas con ánimo de lucro a grand escala, es que estas segundas forman parte de la red de políticas neoliberales que implican a organismos internacionales, empresas, iniciativas filantrópico-capitalistas, edupreuners, académicos, columnistas, gobiernos y grandes corporaciones tecnológicas. A su vez, las EBC 
forman parte de una estrategia de márquetin repensado, que sostiene la idea de que más vale educación asequible a una educación carente.

\section{Notes}

1 Un think tank es una organización privada, sin ánimo de lucro que realiza investigaciones con una función asesora. La influencia de los think tanks en el diseño de políticas públicas en educación está constatada por diversos autores que han estudiado la influencia de estas organizaciones en el diseño de reformas endógenas de privatización de la educación que llevan a preconcebir como la opción más lógica, el aplicar el modelo de Nueva Gestión Pública (Olmedo y Santacruz, 2013).

2 Para más información sobre las escuelas Luminova, consultar: https://www.escolaluminova.com. $\mathrm{br} / \#$ quem-somos

\section{Referencias Bibliográficas}

Adrião, T. (2018). Dimensões e formas da privatização da educação no Brasil: caracterização a partir de mapeamento de produções nacionais e internacionais. Currículo sem Fronteiras, 18 (1), 8-28. Disponible en: http://www.curriculosemfronteiras.org/vol18iss1articles/adriao.pdf

Adrião, T., Garcia, T., Borghi, R., Bertagna, R., Paiva, G., y Ximenes, S. (2016). Grupos empresariais na educação básica pública brasileira: limites à efetivação do direito à educação. Educação e Sociedade, 37 (134), 113-131,

Afridi, M. (2017). Understanding the work of female teachers in Low Fee Private Schools in Punjab, Pakistan. Unpublished doctoral dissertation. OISE: University of Toronto.

Balarin, M. (0216). La privatización por defecto y el surgimiento de las escuelas privadas de bajo costo en el Perú. ¿Cuáles son sus consecuencias? Revista de la Asociación de Sociología de la Educación, 9 (2), 181-196

Ball, S. y Olmedo, A. (2011). Global Social Capitalism: using enterprise to solve the problems of the world. Citizenship, Social and Economics Education, 10 (2-3), 83-90. Doi: https://doi.org/10.2304/ csee.2011.10.2.83

BayBrazil (2017, 11, 29). Chaim Zaher, presidente de Grupo SEB (BayBrazil) [entrevista online]. Disponible en http://www.baybrazil.org/chaim-zaher-president-grupo-seb

Global Campaign for Education (GCE) (2016). Private Profit Public Loss Why the push for low-fee private schools is throwing quality education off track. Johannesburg: Enma Sheery. Disponible en https:// www.campaignforeducation.org

DCI, Diario, Comercio, Industria e Serviço $(2018,07,30)$ Grupo SEB investe em nova rede de escolas para classe C, avalia aquisições após eleição. Disponible en https://www.dci.com.br/servicos/gruposeb-investe-em-nova-rede-de-escolas-para-classe-c-avalia-aquisic-es-apos-eleic-o-1.727739

Grupo SEB. (2019). Grupo SEB-Video Institucional [Video]. Recuperado de https://www.youtube.com/ watch?time_continue=94\&v=xyOfuS1-xV0

Hargreaves, A. (1999). Profesorado, cultura y postmodernidad. Cambian los tiempos, cambia el profesorado. Madrid: Morata. 
Härmä, J. (2011). Low cost private schooling in India: Is it pro poor and equitable? International Journal of Educational Development, 31 (4), 350-356. Doi: https://doi.org/10.1016/j.jedudev.2011.01.003

Instituto Brasileño de Geografía y Estadística (IBGE) (2018). Estimativas da población residente en los municipios brasileños con fecha de referencia en $1^{\circ}$ de julio de 2018.

Klees, S. (2017). Liberia's Experiment with Privatizating Education. National Center for the Study of Privatization in Education, Working Paper 235. Disponible en: http://ncspe.tc.columbia.edu/workingpapers/WP235.pdf

Krippendorf, K. (1997). Metodología de análisis de contenido. Teoría y práctica. Barcelona: Paidós.

Laval y Dardot (2013). La nueva razón del mundo. Ensayo sobre la sociedad neoliberal. Barcelona: Gedisa.

Luengo, J., y Saura, G. (2013). Nuevas formas de mercantilizar la educación. Cuadernos de Pedagogía, 435, 63-65.

Mello, G. (2018). Grupo SEB investe em nova rede de escolas para classe C. Exame. [online] Disponible en: $\quad$ https://exame.abril.com.br/negocios/grupo-seb-investe-em-nova-rede-de-escolas-paraclasse-c/ [Acceso 3 Mar. 2019].

Minayo, M. C. y Costa, A. P. (2018). Fundamentos Teóricos das Técnicas de Investigação Qualitativa. Revista Lusófona de Educação, 40, 139-153. Doi: http://orcid.org/0000-0002-4644-5879

Moschetti, M. (2015). Private education supply in disadvantaged areas of the City of Buenos Aires and 'low-fee private schooling': Comparisons, contexts and implications. Education Policy Analysis Archives, 23(126), 1-26. Doi: http://dx.doi.org/10.14507/epaa.v23.1981

Moschetti, M., Fontdevila, C. y Verger, A. (2017). Manual para el estudio de la privatización de la educación. Bruselas: Internacional de la Educación.

Olmedo, A. y Santa Cruz, E. (2013). Neoliberalism, Policy Advocacy Networks and Think Tanks in the Spanish Educational Arena: The Case of FAES. Education Inquiry, 4 (3), 473-496

Sahlberg, P. (22 de enero de 2012). Global Educational Reform Movement is here! [Mensaje en un blog]. Disponible en: https://pasisahlberg.com/global-educational-reform-movement-is-here/

Siqueira, A. y Nogueira, M. (2017). Focalizando um segmento específico da rede privada de ensino: escolas particulares de baixo custo. Educação e Pesquisa, 43 (4), 1005-1022. Doi: http://dx.doi. org/10.1590/s1517-9702201710177139

Srivastava, P. (2015). Low-fee private schools and poor children: what do we really know?. The Guardian. [online] Disponible en: https://www.theguardian.com/global-development-professionalsnetwork/2015/aug/12/low-fee-private-schools-poverty-development-economist [Acceso 8 Mar. 2019].

Tooley, J., y Dixon, P. (2005). Private Education Is Good for the Poor: A Study of Private Schools Serving the Poor in Low-income Countries. Massachusetts Ave., N.W. Washington, D.C: Cato Institute. 


\section{Cristina Pulido Montes}

Doctoranda en educación en el Departamento de Educación Comparada e Historia de la Educación de la Universidad de Valencia. Sus líneas de investigación se centran en los procesos de neoliberalización de la educación y la privatización de la educación desde una perspectiva comparada.

Email: cristina.pulido@uv.es ORCID: https://orcid.org/0000-0003-1087-461X

\section{Ana Ancheta Arrabal}

Profesora Contratada Doctora en el Departamento de Educación Comparada e Historia de la Educación de la Universidad de Valencia. Sus líneas de investigación son las especializadas en Educación y Atención de la Primera Infancia desde el prisma comparado.

Email: ana.ancheta@uv.es

ORCID: https://orcid.org/0000-0002-9833-242X

Wisllayne Ivellyze de Oliveira-Dri Doctoranda en la Faculdade de Educação de la Universidade Estadual de Campinhas - UNICAMP - (Brasil). Sus líneas de investigación son las temáticas de Políticas Públicas

Educacionales, regionalización y MERCOSUR.

Email: wis_pedagogia@yahoo.com.br ORCID: https://orcid.org/0000-0003-4077-2606

Correspondência

Cristina Pulido Montes

Facultad de Filosofía y Ciencias de la Educación (Universidad de Valencia) Av. de Blasco Ibáñez, 30, 46010 València, Valencia.

Data de submissão: Julho 2019 Data de avaliação: Setembro 2019

Data de publicação: Abril 2020 